\section{Núcleos de Apoio à Saúde da Família: desafios e potencialidades na visão dos profissionais da Atenção Primária do Município de São Paulo, Brasil}

\author{
Family Health Support Centers: challenges and \\ opportunities from the perspective of primary \\ care professionals in the city of São Paulo, Brazil
}

\section{Abstract}

Family Health Support Centers (NASF) were created in Brazil to increase the case-resolution capacity of primary healthcare. Prior to their implementation in the West Side of the city of São Paulo, Brazil, a series of workshops were held for primary healthcare professionals to prepare a proposal for such centers. Hermeneutic analysis was used to study the transcribed material. The thematic categories were: role, constitution, and functioning of the NASF, relationship with family health teams, and interdisciplinarity. The participants' expected the NASF to be an empowering device for comprehensiveness of care, intervening in an existing culture of unnecessary referrals while fostering linkage with other levels of care. The participants also expected the NASF to contribute to the discussion on health professionals' training and stimulating reflection with policymakers on health indicators based exclusively on the number of consultations. These indicators fail to reflect the impact on the services' activities and the quality of care offered to the population in the coverage area.

Primary Health Care; Health Management; Health Personnel; Family Health Program; Qualitative Research
Andréa Tenório Correia da Silva 1

Márcia Ernani de Aguiar ${ }^{2}$

Kelly Winck 2

Karen Gonzaga Walter Rodrigues 2

Mariana Eri Sato 2

Sandra Josefina Ferraz Ellero Grisi 1

Alexandra Brentani 1

Izabel Cristina Rios ${ }^{1}$

\section{Introdução}

O Programa Saúde da Família (PSF) foi criado pelo Ministério da Saúde, em 1994, para promover a reorganização das ações de atenção primária no país. Atualmente, o número de indivíduos assistidos pelas equipes de saúde da família é de 101 milhões, cerca de 52,3\% da população ${ }^{1}$. Para apoiar essas equipes nas atividades relacionadas ao cuidado, o Ministério da saúde criou, em 2008, os Núcleos de Apoio à Saúde da Família (NASF) 2. Cada NASF vincula-se a um número variável de equipes de saúde da família, entre 8 e 20, e pode ser composto por até 19 profissionais 3 , entre eles, estão: psiquiatras, nutricionistas, fisioterapeutas, psicólogos. A implantação dos NASF tem ocorrido de maneira exponencial: em 2008, eram 3 e passaram para 1.498 em 20112.

No Município de São Paulo, a gestão da saúde difere de outras metrópoles do país, uma vez que as equipes de saúde da família e os NASF são gerenciados por instituições parceiras da Secretaria Municipal de Saúde (SMS). Essas são instituições privadas contratadas pela prefeitura e assumem a contratação dos profissionais da atenção primária, assim como, o treinamento, a coordenação e a supervisão direta 4 .

Entre elas está a Fundação Faculdade de Medicina ligada à Faculdade de Medicina da Universidade de São Paulo (FFM-FMUSP), que, a partir de 2008, assumiu o gerenciamento da microrregião Butantã/Jaguaré, que possui cerca de 383 mil 
habitantes. Desse modo, a FFM-FMUSP passou a gerenciar de 14 unidades básicas de saúde (UBS), quatro unidades de atendimento médico ambulatorial e um centro de especialidades ${ }^{5}$. Também se responsabilizou por construir a articulação funcional entre os vários níveis de atenção, isto é, um grau de coordenação e de integração para funcionamento da rede assistencial necessário para assegurar o cumprimento dos princípios e diretrizes do Sistema Único de Saúde (SUS) 6. As ideias que fundamentam o gerenciamento da microrregião são: a integralidade do cuidado, o funcionamento efetivo dos fluxos de referência e contrarreferência para criação de uma plataforma de ensino e pesquisa que contribua para a formação de profissionais de saúde, para a educação permanente dos trabalhadores e que gere conhecimento a partir do referencial da atenção primária à saúde enquanto base organizadora do sistema de saúde. Essa plataforma é chamada de Projeto Região Oeste. Dentre as expectativas que gestores depositam no NASF, está o aumento da resolutividade da atenção primária à saúde vinculado à ideia da potencialidade do trabalho interdisciplinar dos profissionais dos NASF e das equipes de saúde da família e ao melhor acesso aos serviços de saúde pela reorganização da demanda e redução da procura dos serviços da atenção secundária e terciária como porta de entrada no sistema.

Apesar da importância e das expectativas que os NASF vêm assumindo, há notória escassez de literatura sobre o tema. Além disso, diante de implantação do NASF na microrregião Butantã/ Jaguaré, a equipe de coordenação da estratégia saúde da família considerou imprescindível a elaboração de uma proposta de NASF a partir de oficinas de trabalho com os profissionais da atenção primária à saúde, da SMS, os gestores locais, professores universitários e pós-graduandos. Para essa construção, a ideia fundamental era que o NASF deveria atuar de forma integrada às equipes de saúde da família, que estão na região desde 2001, e à rede de serviços local.

Esse manuscrito foi elaborado para descrever a construção coletiva de uma proposta de NASF para a região oeste do Município de São Paulo, com o intuito de compartilhar, com a comunidade científica, os profissionais de saúde e os gestores, experiências que possam contribuir para reflexões sobre o NASF, os desafios da interdisciplinaridade, da integralidade, as práticas ligadas à atenção primária à saúde, e possíveis repercussões na saúde da população.

\section{Método}

A metodologia qualitativa 7 foi escolhida para $o$ estudo por permitir maior aproximação junto às situações de trabalho, possibilitando que fossem discutidas pelo coletivo que as vivenciava no seu cotidiano profissional. Entre diferentes relatos e informações e interpretações sobre interpretações 8 , o estudo das questões de trabalho poderia levar à compreensão mais profunda da realidade em discussão e à maior integração da proposta de NASF com as necessidades percebidas pelos profissionais da atenção primária à saúde e pelos gestores locais.

Entre as técnicas de investigação de questões na metodologia qualitativa, optou-se por realizar oficinas de trabalho ${ }^{9}$ por propiciar campos de intersubjetividade, definida aqui como experiência vivida simultaneamente por várias "mentes", envolvendo testemunho, reconhecimento de subjetividades e empreendimento consciente para entender uns aos outros 10 . Tais oficinas, quando constituídas por pessoas dispostas a aprofundar o conhecimento de um tema, possuem grande potencial para a interação, facilitando alcançar a criação de tal campo de intersubjetividade. Além disso, as oficinas permitem o trabalho com grupos relativamente grandes, tendo, como meta, a elaboração de um "produto" de autoria coletiva.

Como é próprio dessa abordagem de estudo, os participantes das oficinas foram escolhidos mediante critérios qualitativos 7 . Foram convidados profissionais com diferentes características, funções, vivências, formação acadêmica, envolvidas no campo da atenção primária à saúde e motivadas pelo objetivo comum da construção do NASF. Foram eles: médicos, enfermeiros, técnicos de enfermagem, agentes comunitários de saúde, gerentes das unidades básicas de saúde, coordenadores da estratégia de saúde da família, coordenador do NASF, gestores da SMS e profissionais das categorias descritas na Portaria no. 154 2: fonoaudiólogo, fisioterapeuta, terapeuta ocupacional, psiquiatra, psicólogo, nutricionista, acupunturista, pediatra, educador físico, ginecologista, homeopata, assistente social e farmacêutico. Também foram convidados médicos inseridos na atenção primária à saúde e que realizavam matriciamento em 3 unidades básicas de saúde (clínico, pediatra e psiquiatra), além de professores universitários (Medicina, Fonoaudiologia, Fisioterapia e Terapia Ocupacional), residentes de Medicina de Família e Comunidade e aprimorandos em Saúde Coletiva.

As oficinas deste estudo foram planejadas com base nos temas norteadores da interdisciplinaridade e da comunicação, visando ao aprofundamento das principais questões relativas ao NASF, seus contornos estruturais e funcionais, potencia- 
lidades e limites. Para cada uma, definiu-se um moderador e um assistente para a condução das conversas que seguiram o roteiro de perguntas abertas que buscava: (1) identificar os desafios para ampliação do cuidado na atenção primária à saúde; (2) discutir o papel do NASF; (3) estabelecer os princípios norteadores das suas práticas; (4) propor a constituição, ações, atribuições e funcionamento do NASF; (5) delinear relacionamento do conjunto equipes de saúde da família e NASF com outros níveis de atenção.

No início das oficinas foi solicitado o consentimento dos participantes. Em seguida, as discussões foram gravadas, transcritas por terceiros e verificadas quanto à confiabilidade. Foi solicitado parecer do Comitê de Ética em pesquisa da FFMFMUSP que aprovou a divulgação dos resultados da oficina via termo de ciência.

A análise interpretativa hermenêutica 7,9 realizada pelas autoras deste artigo sobre o material transcrito ocorreu de acordo com os seguintes passos: leitura individual e coletiva do materia transcrito, identificação e agrupamento de unidades de significado e construção de categorias de análise temáticas. Foram considerados tanto os consensos quanto os dissensos de todas as intérpretes, tantas vezes quantas fossem necessárias até produzir uma compreensão vinda do campo de conjunção das subjetividades que fizeram parte do estudo, atitude hermenêutica que supõe a existência de opiniões e juízos que devem ser explicitados, qualificados e processados 11 .

As categorias de análise definidas e sobre as quais desenvolvemos o trabalho compreensivo foram: papel do NASF, constituição, funcionamento relação com as equipes de saúde da família e interdisciplinaridade.

\section{Resultados e discussão}

As oficinas de trabalho realizaram-se nos dias 22 23 e 24 de abril de 2009 e contaram com a participação de 24 profissionais divididos em dois grupos.

Em um dos grupos, houve rotatividade dos participantes, assim, optou-se por realizar este estudo com o grupo sem a alternância de pessoas, respeitando as continuidades temporal e temática propostas para as oficinas.

Apresentamos, a seguir, a descrição das oficinas e a análise interpretativa do corpo narrativo constituído pelos vários discursos, aqui, trabalhado na forma de categorias temáticas que constituem o "produto" acabado a posteriori pela interpretação das autoras deste manuscrito sobre os dados produzidos nas oficinas e que formam a estrutura da proposta do NASF.

\section{Descrição das oficinas}

No primeiro dia, foram discutidas as ações das equipes de saúde da família e ampliação do cuidado a partir de um caso de família. Foi solicitado ao grupo que respondesse à pergunta "quais seriam as necessidades de cuidado dessa família?", descrevendo "soluções" possíveis, papéis das equipes de saúde da família e dos profissionais (médico, enfermeira, técnico de enfermagem, agente comunitário de saúde).

No segundo dia, os dois grupos propuseram respostas para as questões: "Que princípios e diretrizes o NASF deve seguir? Qual o papel e constituição do NASF? O que o NASF conseguiria fazer e quais seriam as limitações? Como o conjunto de equipes de saúde da família-NASF se relacionaria com o sistema?".

No terceiro dia, as propostas de NASF foram apresentadas para debate.

\section{Análise interpretativa}

A análise interpretativa das narrativas está disposta em categorias de análise, sendo comentada a seguir.

\section{- Categorias de análise}

\section{a) Papel do NASF}

(i) Potencializar a atenção primária e auxiliar na reorganização da rede de atenção secundária e terciária.

A Portaria $n^{o} .2 .488^{3}$ descreve que, com a responsabilização compartilhada pelo conjunto NASFequipes de saúde da família, o acompanhamento dos casos ocorreria de forma interdisciplinar, e as práticas de encaminhamento seriam reavaliadas, modificando a coordenação do cuidado nas redes de atenção à saúde.

Nesse sentido, o grupo concorda com a portaria ao destacar a necessidade de o NASF potencializar a atenção primária à saúde, ampliar as ações das equipes de saúde da família, articulando as redes de atenção à saúde e, assim, otimizar os fluxos de referência e contrarreferência.

"O primeiro papeléaumentar a resolutividade da atenção primária à saúde e saber determinar os recursos a serem agenciados quando os limites se apresentarem e participar na organização dos outros níveis do sistema".

“...conseguir fazer junto à equipe: potencializar o grau de intervenções e construir novas ações para aumentar a resolutividade...".

"A gente está numa lógica que qualquer equipe multiprofissional que se implante vai se de- 
parar com a potência do trabalho e do território, mas também com os limites de uma rede que não responde. Eu acho que esta equipe deve estimular no território a função das redes".

Uma preocupação destacada pelos participantes foi que o NASF não assuma o papel dos outros níveis de atenção, apesar das filas de espera para atendimento em serviços de atenção secundária. Nesse sentido, Cunha \& Campos 6 afirmam que a falta de serviços de saúde especializados pode conduzir a um funcionamento equivocado do NASF, que assume um papel de "substituição do serviço que falta", na tentativa de responder à demanda da população.

“...uma equipe dessas não tem condição de substituir a rede de saúde...”.

\section{(ii) Reestruturar a atenção à saúde para} reduzir a medicalização

O tema "medicalização" foi debatido pelos participantes da oficina. Barros 12 define medicalização como a dependência dos indivíduos de bens de ordem médico-assistencial, tendo, como característica, a hipervalorização dos medicamentos. Um estudo verificou uma prevalência de consumo de medicamentos de $67,1 \%$ na área de abrangência de uma unidade de saúde da família 13 , e a pesquisa realizada, em 2010, pelo Instituto de Pesquisa Econômica Aplicada (IPEA) 14 verificou que a distribuição gratuita de medicamentos era um dos serviços do SUS mais bem avaliados pela população, e que, para melhorálo, os entrevistados sugeriam aumentar a lista de medicamentos.

A expectativa do grupo foi que o NASF possa atuar como reestruturador da atenção à saúde, formulando ações capazes de dar respostas aos problemas apresentados, agindo no sentido de diminuir a medicalização.

"Uns dos papéis seriam: reestruturar a atenção à saúde a partir da crítica sobre o complexo médico-industrial; definir um plano terapêutico em conjunto com as equipes de saúde da família; criar respostas individualizadas e reduzir a medicalização...".

Vosgerau et al. 13 afirmam que as práticas irracionais de utilização de medicamentos repercutem no aumento da morbimortalidade e dos gastos com saúde. Esses autores destacam que o NASF, atuando de modo integrado às equipes de saúde da família e tendo o farmacêutico, pode contribuir para o uso racional de medicamentos pela população. Essa ideia condiz com a expectativa dos participantes da oficina, mas está em oposição à cultura estabelecida.

\section{(iii) Corresponsabilização do cuidado}

Houve consenso no grupo quanto à corresponsabilização e à definição de um plano terapêutico conjunto também como forma de evitar que o NASF aumente a sobrecarga que as equipes de saúde da família já enfrentam diante das necessidades de saúde da população (cerca de 3.500 pessoas/equipes de saúde da família), da ineficiência das redes de atenção à saúde, das agendas lotadas e da cobrança da gestão por número de consultas.

"O NASF não como solução, mas como parceiro para achar soluções”.

O grupo chama a atenção para a necessidade de repensar a organização do trabalho, as agendas dos profissionais, tanto no que se refere à assistência individualizada quanto para a construção do trabalho interdisciplinar que repercute diretamente na qualidade do cuidado.

"Você pode desperdiçar muito tempo do trabalho das pessoas. Tem que desenvolver um tipo de divisão de trabalho, com ações que são inovadoras para potencializar e integrar ações".

\section{(iv) Atuar no ensino e educação permanente}

Os participantes destacaram a importância de os profissionais do NASF atuarem no ensino. Discutiu-se o tema da inserção de alunos da graduação e da pós-graduação na atenção primária à saúde, além da formação dos profissionais das equipes de saúde da família e do próprio NASF.

“...é como transformar as unidades básicas de saúde que hoje a gente já tem voltadas ao ensino; fazer para que tenham enfermagem, medicina e também fonoaudiologia, fisioterapia e terapia ocupacional".

As atividades do NASF foram vistas como uma possibilidade de ampliação do campo de ensino de diversas especialidades médicas, de enfermagem e de outras categorias profissionais como a terapia ocupacional, fonoaudiologia e fisioterapia. Isso está de acordo com a proposta curricular das Diretrizes Nacionais 15 , que, pautadas nas necessidades do SUS, preconizam a formação de profissionais capazes de atuar na atenção primária à saúde, o que coloca o NASF como potencial espaço de ensino e de pesquisa.

"Por exemplo, para o Residente de Psiquiatria... se um dos mercados de trabalho é NASF, ele tem que ser treinado em NASF".

“...tem que pensar assim: os componentes do NASF têm que ser tutores também... quer dizer, eles vão fazer assistência, ensino e pesquisa...”.

Houve consenso no grupo de que o NASF também atue na Educação Permanente dos 
profissionais da atenção primária à saúde, o que corrobora a Portaria $n^{o} .154^{2}$. Entretanto, foi colocada a necessidade de o próprio profissional do NASF ser formado em campo, já que, boa parte das vezes, ele não terá experiência em atenção primária à saúde. Segundo Barbosa et al. 16, há necessidade de adequação da formação às propostas do SUS e às diversas atribuições dos profissionais estabelecidas não apenas pela teorização das características do profissional na atenção primária à saúde, mas também de acordo com as necessidades de saúde da população.

"Este profissional que a gente quer no NASF tem que se formar em campo". "O NASF ninguém aprendeu na escola".

Nascimento \& Oliveira 17 descrevem que, atualmente, dentre as dificuldades reconhecidas no processo de trabalho do NASF, está a formação dos profissionais, que não é compatível com as necessidades do SUS. As propostas pedagógicas são construídas distanciadas dos serviços e não contemplam pontos chaves como o trabalho em equipe, o vínculo, o acolhimento, imprescindíveis para o NASF.

\section{b) Constituição do NASF}

A Portaria $n^{o} .2 .488$ define que os profissionais que podem compor o NASF são: médico acupunturista; assistente social, educador físico, farmacêutico, fisioterapeuta, fonoaudiólogo, ginecologista, homeopata, nutricionista, pediatra, psicólogo, psiquiatra, terapeuta ocupacional, geriatra, clínico geral, médico do trabalho, veterinário, arte-educador e um sanitarista ${ }^{3}$. Entretanto, o grupo enfrentou muita dificuldade para propor a composição do NASF e o número de cada profissional, sobretudo quando se tratava de inserir profissional não médico.

“Nesta questão dos princípios que ajudam a gente a nortear quem compõe é pensar de que maneira a gente lê os grupos de pessoas que estão cadastrados. As demandas...".

Nesse sentido, Pires et al. 18 afirmam que é necessário conhecer a oferta e a demanda dos serviços de saúde locais para subsidiar a formulação de políticas públicas. Em vez de criar demandas a partir da oferta indiscriminada e desigual de serviços, esses é que devem se estruturar a partir das exigências dos usuários.

Apesar de ter sido consenso no grupo que os profissionais do NASF devem atender às demandas existentes, houve dificuldade em delinear as reais necessidades da comunidade. Essa discussão sobre oferta de serviços, demandas e necessidades de saúde foi destacada pelos participantes da oficina.
"A universalização é dada pela oferta de serviços e não necessariamente para a leitura que a gente constrói de quem mora na região".

"O NASF tem que compor com aquilo que já é demanda dos serviços e aquilo que na universalização da atenção não pode ser ainda cuidado".

Para Schraiber \& Mendes-Gonçalves 19, o conceito de necessidade de saúde eleito pelos serviços tem sido o operacional, associado ao consumo de um serviço, geralmente a consulta médica. Para Sucupira \& Ferrer 20, as demandas específicas são determinadas pela proposta de atendimento dos serviços de saúde.

$\mathrm{Na}$ oficina, as falas que se referem à composição do NASF também estão relacionadas às vivências dos participantes com o matriciamento que acontece na região oeste. Assim, para os profissionais médicos do NASF, o grupo definiu uma proporção de UBS por profissional e a carga horária. Para outras categorias profissionais, o grupo evidenciou dissenso.

“...em relação aos especialistas médicos ter-seia 1 psiquiatra, 1 clínico, 1 pediatra e 1 ginecoobstetra para cada 3 unidades, com a carga de 20 horas semanais".

"...A gente ainda não falou de alguns profissionais, como a assistente social... Alguém chuta um número?".

Para coordenar as equipes do NASF, haveria um gerente com experiência em gestão e atenção primária à saúde.

A seleção dos profissionais do NASF também foi pontuada, principalmente no sentido de definir como selecionar, que qualificações os profissionais deveriam apresentar, incluindo a compreensão dos princípios da atenção primária à saúde.

"Há dificuldade de se pensar a seleção dessas pessoas. Como selecionar e pactuar o papel dos profissionais que vão chegar; pensar em quem ele é, não só qual tipo de profissional".

\section{c) Funcionamento do NASF}

O grupo debateu a necessidade de uma definição inicial do processo de trabalho a partir das necessidades do território e articulado às atividades das equipes de saúde da família. Entretanto, foi consenso que o formato não seja "engessado" para que, de acordo com a sua realidade e demanda, o NASF possa ter autonomia de articular com as equipes de saúde da família, e identificar as prioridades e a maneira de atuação.

"Tem que estar à prova todo o tempo porque ninguém tem resposta pra isso".

"Organizar um dispositivo que dê respostas às demandas descentralizadas, individualizadas. Então como dimensionar?". 
A SMS de São Paulo ${ }^{21}$ propõe que o NASF atue realizando as seguintes atividades: atendimentos específicos ( $5 \%$ a $30 \%$ da carga horária mensal), ações compartilhadas (35\% a $80 \%)$ e de ações intersetoriais (5\% a 10\%). Nesse sentido, o grupo destaca que as atividades do NASF devem incluir: reuniões com as equipes de saúde da família e da UBS, matriciamento, visitas domiciliares, atendimento compartilhado. Houve discussão quanto à assistência individual.

O grupo ressaltou a potencialidade das reuniões para discussão dos casos e o planejamento das ações.

"As reuniões são um fórum legítimo para as discussões. Os profissionais têm que entrar nesses fóruns e participar, para discutir entre si as ações conjuntas e construir".

"Eles têm de participardas reuniões das unidades, em que se discutem principalmente os fluxos de trabalho. Traça principalmente a filosofia".

Para Cunha \& Campos 6, o apoio matricial é uma ferramenta tecnológica que oferece retaguarda assistencial e suporte técnico-pedagógico às equipes de referência e promove o compartilhamento dos saberes, ampliando a resolução dos problemas. O apoio matricial se propõe a alterar a lógica de encaminhamentos indiscriminados para uma lógica de corresponsabilização territorial 22, buscando maior resolutividade para assegurar, de forma dinâmica e interativa, retaguarda especializada às equipes.

$\mathrm{O}$ modelo de matriciamento que ocorre na microrregião Butantã/Jaguaré é de caráter técnico-pedagógico, feito por profissionais médicos com experiência profissional de vários anos na atenção primária à saúde junto às equipes de saúde da família e aos residentes de Medicina de Família e Comunidade. A discussão no grupo aconteceu diante desse contexto.

“O nutricionista, por exemplo, é uma das áreas com maior nitidez de como vai acontecer o matriciamento".

“...não pode ter só matriciamento, porque chega uma hora que você esgota todas as possibilidades... tem que ter o atendimento também".

Com relação a consulta individual enquanto ação do NASF, o grupo manifestou preocupação com o risco de que essa atividade assuma o papel da atenção secundária, embora o grupo reconheça que esse recurso possa ser indispensável para aumentar a compreensão do caso e potencializar a resolutividade.

"Tem que assumir junto o rojão dessa deman$d a ”$.

"Ele [NASF] entra nas situações em que o secundário seria necessário".

"Não acho! Se não a gente volta no risco dele virar uma tentativa de secundário”.
Nascimento \& Oliveira 17 (p. 94) postulam que o NASF tem como principal desafio alterar a cultura organizacional no SUS, “...que prioriza a quantidade em detrimento da qualidade, o referenciamento em detrimento da resolutividade e a avaliação de impacto e de indicadores de saúde por ações meramente quantitativas, em detrimento das qualitativas...". Para esses autores, o NASF, tendo como base a clínica ampliada, visa melhorar a qualidade do serviço ofertado e não apenas suprir a demanda assistencial no aspecto numérico. O funcionamento do NASF tem, como desafio, a articulação das suas atividades dentro da agenda das equipes de saúde da família, considerando as metas assistenciais e o processo de trabalho já estabelecido.

Houve dissenso no grupo em relação à possibilidade de o usuário ter acesso direto ao NASF sem passar pela equipe de saúde da família. Alguns participantes defenderam que os indivíduos devem ser direcionados para consulta individual ou compartilhada apenas após a discussão do caso com a equipe de saúde da família. Outros afirmaram que deveria existir uma possibilidade de agendamento direto com o profissional do NASF via grupos de promoção da saúde ou pelo acolhimento.

"Sem dúvida a porta de entrada vai ser sempre mais a equipe de saúde da família...”.

"...mas acho que algumas outras portas têm que estar espalhadas (...) alguns grupos devem ser de acesso livre ao NASF".

\section{d) Relação com Estratégia Saúde da Família e interdisciplinaridade}

A discussão no grupo sobre a relação entre os profissionais da equipes de saúde da família e do NASF foi extensa. Ao mesmo tempo em que seria necessário privilegiar o núcleo dos saberes como portador de conhecimentos específicos, ressaltou-se a importância de evitar a hierarquização, o especialismo e a fragmentação, buscando uma relação dialógica e interdisciplinar.

"Fazer com que não seja uma relação verticalizada, a do NASF com os profissionais da UBS".

"...levantar o conhecimento específico de cada um, mas não segmentando a atenção à pessoa...”.

“...medo de segmentar o cuidado com a chegada do NASF...”.

Mendes et al. 23 (p. 31) descrevem a interdisciplinaridade como "a interação dinâmica entre os saberes" e como ponto auxiliar do processo de trabalho e a efetividade do cuidado, na medida em que diferentes conceitos podem interagir.

O grupo concorda que, na fase inicial do NASF, deve ocorrer a soma de conhecimentos isolados, já que os profissionais não têm ainda 
experiência sobre essa realidade, assim, a relação será multidisciplinar. A expectativa é que, com o passar do tempo, havendo uma crescente apropriação dos casos, das características do território e integração com as equipes de saúde da família, será possível a construção de novos saberes que transitarão pelas diversas especialidades de maneira dinâmica, indo em direção à transdisciplinaridade.

“...como alcançar a transdisciplinaridade?... uma vez que isso não está dado a priori, será processo de construção...”.

Para o êxito da interdisciplinaridade, devem existir um objetivo comum e a disponibilidade ao diálogo ${ }^{6}$. Essa troca de conhecimentos dialógica é fundamental para as práticas de saúde, e bastante evidente na atenção primária à saúde, na qual ninguém é detentor de todo o saber necessário. Segundo Morin 24 (p. 138), “o problema da complexidade não éo da completude, mas o da incompletude do conhecimento".

O grupo explicita claramente a preocupação de como a interdisciplinaridade acontecerá na prática e como a interação se estabelecerá entre esses vários profissionais com os seus diferentes saberes.

Nesse sentido, Campos \& Dominitti 25 afirmam que a equipe de referência deve considerar a complexidade da atenção primária à saúde e a interdependência entre os profissionais, principalmente. Desse modo, a coordenação de uma equipe de referência deve estar direcionada a criar a condição necessária para haver integração entre os profissionais para que busquem projetos comuns. Enxergar as diferenças existentes entre os profissionais, tentando aproveitá-las para resolução dos problemas, para a construção dos projetos terapêuticos e das intervenções.

\section{Considerações finais}

A ESF é responsável pelo cuidado de aproximadamente 101 milhões de pessoas. Desde a sua criação em 1994, lida com as limitações de um sistema de saúde que, muitas vezes, fornece respostas aquém das necessidades de saúde da população. É nesse contexto que o NASF foi criado, em 2008, pelo Ministério da Saúde, visando apoiar as ações das equipes de saúde da família e contribuir para ampliar a resolutividade da atenção primária à saúde, buscando a integralidade do cuidado, tendo, como lócus principal de desenvolvimento, as ações da atenção primária à saúde e das equipes de saúde da família.

A política de educação permanente do Ministério da Saúde 26 (p. 22) postula que "trabalhadores e gestores participem das discussões sobre as necessidades sociais concretas no trabalho, desde diagnóstico de situação e demandas, inclusive as educacionais, até a construção coletiva de propostas para gestão e organização dos processos de trabalho". Essa política contribuiu para que a equipe de coordenação das equipes de saúde da família da microrregião Butantã/Jaguaré propusesse a realização das oficinas. Além disso, foram fatores que estimularam a realização das oficinas o fato de haver pouca experiência com o NASF no contexto nacional e, principalmente, a necessidade prática de implantá-lo na região oeste do Município de São Paulo.

A realização das oficinas partiu do pressuposto de que o trabalho na área da saúde não pode ser um simples "executar tarefas", mas um constante pensar sobre o trabalho capaz de criar estratégias que recuperam significados tanto para os usuários quanto para os profissionais. No pensar coletivo, são definidos nós críticos ou pontos chaves; conhecimento e compreensão relativos aos mesmos são buscados; levantam-se hipóteses de solução e desenham-se planos de ação que podem ser aplicados à realidade. Definem-se necessidades e respostas, e gestão participativa e humanização são feitas 27.

Os achados das oficinas realizadas neste estudo evidenciaram a expectativa de que o NASF seja um dispositivo inovador que possa potencializar as ações das equipes de saúde da família e confrontar os desafios a serem enfrentados, como: a corresponsabilidade pelo cuidado, a interdisciplinaridade sem descaracterizar as especificidades, a articulação funcional entre os diversos níveis de atenção, em especial com o nível secundário, a garantia de espaços nas agendas das equipes de saúde da família e do NASF para discussão de casos, a elaboração do plano de cuidados e ações compartilhadas e o papel do NASF como formador de recursos humanos.

O NASF foi identificado pelos participantes da oficina como um dispositivo fundamental para potencializar a integralidade do cuidado, a resolutividade da atenção primária à saúde e também do SUS, intervindo na cultura dos encaminhamentos desnecessários, promovendo a discussão da formação dos profissionais de saúde. O NASF também contribuiria para evidenciar os nós críticos do sistema de saúde. Para tanto, há necessidade de estar integrado às equipes de saúde da família, com interação dos núcleos de saberes de maneira dinâmica e para garantir a construção do plano de cuidado sem fragmentação.

Um ponto importante ressaltado pelos participantes da oficina foi que o NASF venha contribuir para a discussão junto aos gestores sobre os indicadores de saúde, fundamentalmente indicadores que evidenciem a qualidade do cuidado 
prestado à população, e para gerar uma reflexão sobre a ineficácia da manutenção da cultura organizacional no SUS de valorização de indicadores estabelecidos por metas meramente quantitativas ligadas ao número de atendimentos, que não refletem o impacto das ações desenvolvidas e são insuficientes para avaliar a qualidade do cuidado. Os indicadores de saúde vinculados à qualidade do cuidado devem evidenciar a garantia do acesso, a resolutividade e, principalmente, a integralidade. Discutir esses indicadores é um desafio a ser enfrentado pelos gestores e pelos profissionais de saúde. A mensuração dos resultados obtidos pelas ações de saúde e a verificação do cumprimento dos objetivos propostos pelo sistema são uma tarefa a ser aprimorada.

Diante do caráter inovador, dos desafios estabelecidos pela realidade da saúde no país e das expectativas relacionados aos NASF, tornase imprescindível a realização de pesquisas que abordem esse tema e contribuam para ampliar as discussões sobre a concepção, o funcionamento e a avaliação do impacto das ações do NASF na qualidade do cuidado.

\section{Resumo}

Os Núcleos de Apoio à Saúde da Família (NASF) foram criados para ampliar a resolutividade da atenção primária. A iminência da implantação na região oeste do Município de São Paulo, Brasil, motivou a realização de oficinas para elaborar uma proposta de NASF por profissionais da atenção primária à saúde. Utilizamos a análise hermenêutica para estudar o material transcrito. As categorias temáticas foram: papel, constituição, funcionamento, relação com a equipes de saúde da família e interdisciplinaridade. A expectativa dos participantes foi de que o NASF seja um dispositivo potencializador da integralidade do cuidado, intervindo na cultura dos encaminhamentos desnecessários e na articulação com os outros níveis de atenção; além de contribuir para a discussão da formação dos profissionais e de estimular a reflexão junto aos gestores sobre indicadores de saúde vinculados exclusivamente ao número de atendimentos, que não refletem o impacto das ações desenvolvidas nem a qualidade do cuidado oferecido à população adscrita.

Atenção Primária à Saúde; Gestão em Saúde; Pessoal de Saúde; Programa Saúde da Família; Pesquisa Qualitativa

\section{Colaboradores}

A. T. C. Silva contribuiu na concepção, desenho do estudo, coleta dos dados, levantamento bibliográfico, análise e interpretação dos dados, escrita e revisão do texto. M. E. Aguiar colaborou na concepção, levantamento bibliográfico, coleta dos dados, análise e interpretação dos dados e escrita e revisão do manuscrito. K. Winck participou no levantamento bibliográfico, coleta de dados, análise e interpretação dos dados, incluindo escrita e revisão do manuscrito. K. G. W. Rodrigues e M. E. Sato contribuíram no levantamento bibliográfico, análise e interpretação dos dados e revisão do texto. S. J. F. E. Grisi participou na análise dos dados e revisão do artigo. A. Brentani colaborou na concepção, análise e interpretação dos dados e revisão do artigo. I. C. Rios contribuiu na concepção, análise e interpretação dos dados, metodologia e revisão do artigo.

\section{Agradecimentos}

A todos aqueles que participaram das oficinas: aos profissionais da Estratégia Saúde da Família da região oeste do Município de São Paulo, da Secretaria Municipal de Saúde, aos professores da Faculdade de Medicina da Universidade de São Paulo, em especial do Departamento de Fonoaudiologia, Fisioterapia e Terapia Ocupacional, e aos matriciadores Lygia Maria de França Pereira, Rodrigo Diaz Olmos, Sandra Callioli Zuccolotto e Wagner Ranna, aos residentes de Medicina de Família e Comunidade e aos aprimorandos em Saúde Coletiva. À Mariana Maleronka Ferron pela contribuição na coleta dos dados, análise e interpretação dos dados. Agradecemos em especial aos Professores Ricardo Rodrigues Teixeira e José Ricardo de Carvalho Mesquita Ayres pelas valiosas discussões sobre comunicação em saúde e interdisciplinaridade, temas fundamentais e norteadores das oficinas. 


\section{Referências}

1. Ministério da Saúde. Atenção básica e saúde da família. http://189.28.128.99/dab/abnumeros. php\#mapas.pdf (acessado em 20/Abr/2012).

2. Ministério da Saúde. Portaria no. 154, de 24 de janeiro de 2008. Cria os Núcleos de Apoio à Saúde da Família NASF. Diário Oficial da República Federativa do Brasil 2008; 25 jan.

3. Ministério da Saúde. Portaria GM nº. 2488, de 21 de outubro de 2011. Aprova a Política Nacional de Atenção Básica, estabelecendo a revisão de diretrizes e normas para a organização da Atenção Básica, para a Estratégia Saúde da Família (ESF) e o Programa de Agentes Comunitários de Saúde (PACS). Diário Oficial da União 2011; 24 out.

4. Bousquat A, Cohn A, Elias PEA. Implantação do Programa Saúde da Família e exclusão socioespacial no Município de São Paulo, Brasil. Cad Saúde Pública 2006; 22:1935-43.

5. Brentani AV, Silva ATC, Dourado AD, Santos IS, Eluf Neto J, Elias PEM, et al. Cadernos Região Oeste: condições socioeconômicas e de saúde. São Paulo: Faculdade de Medicina, Universidade de São Paulo; 2009.

6. Cunha GT, Campos GWS. Apoio matricial e atenção primária em saúde. Saúde Soc 2011; 20 : 961-70.

7. Minayo MCS. O desafio do conhecimento. Pesquisa qualitativa em saúde. 3a Ed. São Paulo: Editora Hucitec/Rio de Janeiro: ABRASCO; 1994.

8. Geertz C. Interpretação das culturas. Rio de Janeiro: Jorge Zahar Editor; 1978.

9. Schraiber L. Pesquisa qualitativa em saúde: reflexões metodológicas do relato oral e produção de narrativas em estudo sobre a profissão médica. Rev Saúde Pública 1995; 29:63-74.

10. Bernstein RJ. Beyond objectivism and relativism: science, hermeneutics and praxis. Philadelphia: University of Pennsylvania Press; 1983.

11. Gadamer H-G. O mistério da saúde? O cuidado da saúde e a arte da Medicina. Lisboa: Edições 70; 2002.

12. Barros JAC. Pensando o processo saúde doença: a que responde o modelo biomédico? Saúde Soc 2002; 11:67-84.

13. Vosgerau MZS, Soares DA, Sousa RKT, Matsuo T, Carvalho GS. Consumo de medicamentos entre adultos na área de abrangência de uma Unidade de Saúde da Família. Ciênc Saúde Coletiva 2011; 16:1629-38.

14. Instituto de Pesquisa Econômica Aplicada. Sistema de indicadores de percepção social. http:// www.ipea.gov.br/portal/images/stories/PDFs/ SIPS/110207_sipssaude.pdf (acessado em 20/ Mar/2011)

15. Conselho Nacional de Educação, Ministério da Educação. Diretrizes curriculares nacionais dos cursos de graduação em enfermagem, medicina e nutrição.http://portal.mec.gov.br/dmdocuments/ ces1133.pdf (acessado em 20/Mar/2011).
16. Barbosa EG, Ferreira DLS, Furbino SAR, Ribei ro EEN. Experiência da fisioterapia no Núcleo de Apoio à Saúde da Família em Governador Valadares. Fisioter Mov 2010; 23:323-30.

17. Nascimento DDG, Oliveira MAC. Reflexões sobre as competências profissionais para o processo de trabalho nos Núcleos de Apoio à Saúde da Família. Mundo Saúde (Impr.) 2010; 34:92-6.

18. Pires MRGM, Gottems LBD, Martins CMF, Guilhem D, Alves ED. Oferta e demanda por média comple xidade/SUS: relação com atenção básica. Ciênc Saúde Coletiva 2010; 15:1009-19.

19. Schraiber LB, Mendes-Gonçalves RB. Necessidades de saúde e atenção primária. In: Schraiber LB, Nemes MIB, Mendes-Gonçalves RB, organizadores. Saúde do adulto na unidade básica. 2a Ed. São Paulo: Editora Hucitec; 1996. p. 29-47.

20. Sucupira ACS, Ferrer APS. Uma experiência de ensino de propedêutica pediátrica em ambulatório. Pediatria (São Paulo) 2000; 22:105-12.

21. Coordenação da Atenção Básica, Secretaria de Saúde, Prefeitura de São Paulo. Diretrizes e parâmetros norteadores das ações dos Núcleos de Apoio a Saúde da Família. http://www.prefeitura. sp.gov.br/cidade/secretarias/upload/saude/ar quivos/esf/diretrizes_nasf.pdf (acessado em 20/ Mai/2010).

22. Departamento de Atenção Básica, Secretaria de Atenção à Saúde, Ministério da Saúde. Diretrizes do NASF: Núcleo de Apoio à Saúde da Família. http://189.28.128.100/dab/docs/publicaco es/cadernos_ab/abcad27.pdf (acessado em 10/ Out/2010).

23. Mendes RMJ, Lewgoy AMB, Silveira EC. Saúde e interdisciplinaridade: mundo vasto mundo. Revista Ciência \& Saúde 2008; 1:24-32.

24. Morin E. Ciência com consciência. 8ạ Ed. Rio de Janeiro: Berthand Brasil; 1999.

25. Campos GWS, Dominitti AC. Apoio matricial e equipe de referência: uma metodologia para ges tão do trabalho interdisciplinar em saúde. Cad Saúde Pública 2007; 23:399-407.

26. Ministério da Saúde. A educação permanente entra na roda: polos de educação permanente em saúde. http://bvsms.saude.gov.br/bvs/publica çoes/educacao_permanente_entra_na_roda.pdf (acessado em 20/Out/2010)

27. Rios IC. Caminhos da humanização - prática e reflexão. São Paulo: Áurea; 2009

Recebido em 03/Jul/2011

Versão final reapresentada em 24/Abr/2012

Aprovado em 13/Jul/2012 